Modeling the Pulse Line Ion Accelerator (PLIA): an algorithm for quasi-static field solution.

A. Friedman, R. J. Briggs, D. P. Grote, E.

Henestroza, W. L. Waldron

June 20, 2007

2007 Particle Accelerator Conference

Albuquerque, NM, United States

June 25, 2007 through June 29, 2007 
This document was prepared as an account of work sponsored by an agency of the United States Government. Neither the United States Government nor the University of California nor any of their employees, makes any warranty, express or implied, or assumes any legal liability or responsibility for the accuracy, completeness, or usefulness of any information, apparatus, product, or process disclosed, or represents that its use would not infringe privately owned rights. Reference herein to any specific commercial product, process, or service by trade name, trademark, manufacturer, or otherwise, does not necessarily constitute or imply its endorsement, recommendation, or favoring by the United States Government or the University of California. The views and opinions of authors expressed herein do not necessarily state or reflect those of the United States Government or the University of California, and shall not be used for advertising or product endorsement purposes. 


\title{
Modeling the Pulse Line Ion Accelerator (PLIA): an algorithm for quasi-static field solution*
}

\author{
A. Friedman ${ }^{\dagger 1,2}$, R.J.Briggs ${ }^{4}$, D.P.Grote ${ }^{1,2}$, E.Henestroza ${ }^{1,3}$, and W.L. Waldron ${ }^{1,3}$ \\ ${ }^{1}$ Heavy Ion Fusion Science Virtual National Laboratory; ${ }^{2}$ LLNL, Livermore, CA; \\ ${ }^{3}$ LBNL, Berkeley, CA; ${ }^{4}$ SAIC, Alamo CA
}

\begin{abstract}
The Pulse-Line Ion Accelerator (PLIA) is a helical distributed transmission line. A rising pulse applied to the upstream end appears as a moving spatial voltage ramp, on which an ion pulse can be accelerated. This is a promising approach to acceleration and longitudinal compression of an ion beam at high line charge density. In most of the studies carried out to date, using both a simple code for longitudinal beam dynamics and the Warp PIC code, a circuit model for the wave behavior was employed; in Warp, the helix I and V are source terms in elliptic equations for $\mathrm{E}$ and B. However, it appears possible to obtain improved fidelity using a "sheath helix" model in the quasi-static limit. Here we describe an algorithmic approach that may be used to effect such a solution.
\end{abstract}

\section{THE MODEL}

In this note we present an algorithm for solving for the fields in a PLIA [1, 2, 3, 4, 5], via an "almost first principles" model [1] which accounts for mutual capacitances and inductances and can capture end effects, transformer coupling, and dispersion. This is an intermediate level of description, between a simple circuit model [6] and a full electromagnetic field computation [7]. Field detail near the helix wires is not captured, but for beam simulation purposes it is not generally needed. Other recent PLIA research is also described these Proceedings $[8,9]$ and elsewhere [10].

In the axisymmetric limit, charge continuity requires:

$$
\frac{\partial \lambda(z, t)}{\partial t}=-\frac{\partial I(z, t)}{\partial z}
$$

where $\lambda(z, t)$ is the charge per unit axial length. The helix current $I(z, t)$ can be thought of as charge per unit time passing through a cross-sectional plane cut across the wire itself, or (equivalently) through a plane cut across the helix and normal to its major axis. Denoting the helix radius by $a$ and the wire center-to-center spacing by $s$, the surface charge density on the helix sheet $\left[\mathrm{Coul} / \mathrm{m}^{2}\right]$ is $\sigma=\lambda / 2 \pi a$, the azimuthal sheet current is $K_{\theta}=I / s[\mathrm{Coul} / \mathrm{m} / \mathrm{s}]$, and the axial sheet current is $K_{z}=I / 2 \pi a[\mathrm{Coul} / \mathrm{m} / \mathrm{s}]$. Eq. 1 can be rewritten as:

$$
\frac{\partial \sigma(z, t)}{\partial t}=-\frac{\partial K_{z}(z, t)}{\partial z} .
$$

* Work performed under auspices of U.S. DoE by the Univ. of CA, LLNL and LBNL under Contract Nos. W-7405-Eng-48 and DE-AC02-05CH11231.

$\dagger$ af@1lnl.gov
We will use Eq. 2 to advance the surface charge density $\sigma(z)$ to the future time level, and then compute the advanced-time electrostatic potential $\phi(r, z)$, and thus the electric field $E(r, z)$ that is needed in the interior of the helix to advance the simulation particles.

It is now possible to establish a correspondence with the circuit model of [6]; assume (for convenience) one computational node per turn of the helix, that is, $\Delta z=s$. The $\sigma_{i}$ at each node $i$ can be integrated around the helix circumference and over an axial interval $\Delta z$ to yield a charge $Q_{i}$. Then, those charges are related to the voltages on the set of nodes by the mutual capacitances:

$$
\sum_{j} C_{i j} V_{j}=Q_{i}
$$

In the simplest model, the only nonzero elements of $C_{i j}$ were the diagonal entries. Here we may precompute the capacitance matrix $C_{i j}$ and, at each time step, invert it to obtain the advanced-time voltages after we have computed the source terms via Eq. 2. In the circuit model, the capacitance between each helix turn and the grounded outer pipe (with dielectric in between) plays a major role. Since $\sigma$ only establishes the jumps in $E_{r}$ and not the actual potential values, the outer-wall boundary condition in the Poisson solution used to establish $C_{i j}$ serves to set the capacitances to ground, $C_{i i}$.

For the purposes of a 1-D circuit model, knowledge of the capacitance matrix suffices. However, to advance the particles in an $(r, z)$ or 3-D simulation it is necessary to obtain the electric field structure, and so (rather than using the capacitance matrix) a Poisson solution may be carried out at each step, including the beam charge as a source term.

The helix voltage $V(z, t)$ is related to the changing axial magnetic flux through the helix. Taking a path of integration in Faraday's law that passes inside the helix wire, extending axially by a distance $\Delta z$, the flux $\Phi(z, t)$ is encircled $\Delta z / s$ times; the corresponding voltage change is:

$$
\Delta V=-\frac{\Delta z}{s} \frac{\partial}{\partial t} \Phi(z, t),
$$

where the total flux through the helix (due to its own current and that of any driving "primary" winding) is:

$$
\Phi(z, t)=\int_{0}^{a} B_{z}(r, z, t) 2 \pi r d r .
$$

In the continuum limit,

$$
\frac{\partial}{\partial t} \Phi(z, t)=-s \frac{\partial V(z, t)}{\partial z} .
$$


It is the magnetic field $\mathbf{B}(r, z, t)$, not the flux, that is desired at the advanced time, and initially it was not clear that the flux (a scalar function of $z$ ) contains sufficient information to uniquely specify $\mathbf{B}(r, z, t)$ (a vector function of $r$ and $z$ ). A key realization was that a set of $N$ fluxes can uniquely specify a set of $N$ current sources (corresponding to $N$ turns of the helix, when $\Delta z=s$ ), and then those sources can be used to compute $\mathbf{B}(r, z, t)$.

The azimuthal magnetic field component, $B_{\theta}(r, z)$, is neglected; as seen in direct solutions of the Maxwell equations [7], it is small near the beam. Thus the current sources are assumed purely azimuthal (circular hoops); at each computational node on the helix, the source is $K_{\theta}(z, t) \Delta z=(2 \pi a / s) K_{z}(z, t) \Delta z=I(z, t) \Delta z / s$.

To make correspondence with the above-mentioned circuit model, the voltage change from node $i$ to node $i+1$ is related to the currents at the nodes by:

$$
\Delta z \sum_{j} M_{i j} \frac{d K_{\theta}}{d t}=\frac{\Delta z}{s} \sum_{j} M_{i j} \frac{d I_{j}}{d t}=V_{i+1}-V_{i}
$$

In the circuit model, $I_{i}$ is the current flowing between "voltage" nodes $i$ and $i+1$. It is desirable to avoid two-cell difference expressions such as $V_{i+1}-V_{i-1}$. The Yee discretization of finite-difference time-domain electromagnetics [11] employs "staggered" grids for $E$ and $B$, so as to preserve important properties of the continuum equations. Thus, we "offset" the axial locations of the currents from those of the voltages by half a cell. By examination of the governing equations, the "centering" of all other quantities can be developed. See Fig. 1.

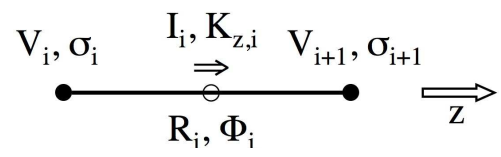

Figure 1: Discretization, showing computational nodes.

When $\Delta z=s$, Eq. 7 describes the usual mutualinductance relationship. Most of our work with the circuit model used the simpler local self-inductance coupling, $L_{i}=-M_{i i}$. For any node spacing, equations 6 and 7 are equivalent (in a finite-difference sense) when:

$$
\sum_{j} M_{i j} I_{j}=-\Phi_{i}
$$

which is a familiar formula for mutual inductances [12]. In practice, the $M_{i j}$ are pre-computed by setting $I_{j}$ to unity for each $j$ in turn, with the other currents zero, computing $\mathbf{B}(r, z)$, and measuring the fluxes $\Phi_{i}$ at all axial nodes.

The model does not require $\Delta z=s$. To allow arbitrary zoning in the simulation code, we interpret the mutual inductances as coupling the magnetic fluxes through the tori associated with the individual computational zones with the currents in those zones. Note that we associate $I_{j}$ with the current flowing in the helix wire through the plane $z=z_{j}$.
$I_{j}$ is not the total current flowing azimuthally in computational zone $j$ on the helix, and $i$ insensitive to zone size when sufficiently small zones are employed. To avoid confusion on this point, we rewrite Eq. 8 as:

$$
2 \pi a \sum_{j} M_{i j} K_{z, j}=-\Phi_{i},
$$

\section{AN ALGORITHM}

Many variations are available; we outline one possibility. The algorithm consists of a pre-computation phase to establish matrices that are needed during the time-advance, and a series of actions at each computational time step, to advance the system through an interval $\Delta t$. Denoting the time level (abbreviated "tll") of a quantity by a superscript, the step is described herein as an advance of the system from tl 0 to tl 1 . The sheet current $K_{z}$ is advanced from tl $1 / 2$ to tl $3 / 2$; it can be obtained at tl 1 by interpolation for diagnostics or magnetic field computation. The overall procedure is formally a "leap-frog" advance, and is "time centered," reversible, and second-order accurate. At startup, the current in the helix may be assumed to be zero, or a half-step may be taken to obtain values of $K_{z}$ at tl $1 / 2$.

Pre-computation: If only the voltages and currents on the helix are desired (an improved circuit model), precomputation of the capacitance matrix is appropriate; it may be desirable in general. An enhanced 1-D particle model might use applied fields computed via a separately precomputed Green's function that relates a unit voltage on the helix at $z=z_{0}$ to a voltage pattern $V\left(z-z_{0}\right)$ averaged over a nominal beam cross-section. Beam self forces might be modeled using a simple " $d \lambda / d z$ " formulation, or (better) yet another precomputed Green's function averaging across a nominal beam cross-section for both the charge-density and the force on a "slice" as a function of $z$. In all cases, the mutual inductances of Eq. 9 should be pre-computed.

Time advance: Enter the time step with $\sigma$ defined at tl 0 and $K_{z}$ defined at tl $1 / 2$.

(a) Advance $\sigma$ using the continuity equation:

$$
\left(\sigma_{i}^{1}-\sigma_{i}^{0}\right) / \Delta t=-\left(K_{z, i}^{1 / 2}-K_{z, i-1}^{1 / 2}\right) / \Delta z .
$$

(b) For the simplest case of an improved circuit model, it suffices to associate charges $Q_{i}=\sigma_{i} \Delta z$ with each node, and solve Eq. 3 for the $V_{j}$ values. For the full simulation case, the surface charge induces a jump in $E_{r}$ at the helix:

$\epsilon_{\text {out }}\left[\frac{\partial \phi^{1}(r, z)}{\partial r}\right]_{r=a^{+}}-\epsilon_{\text {in }}\left[\frac{\partial \phi^{1}(r, z)}{\partial r}\right]_{r=a^{-}}=-\sigma^{1}(z)$,

where $\epsilon$ is the dielectric constant, and "out" and "in" denote $r>a$ and $r<a$. There are multiple possibilities:

(i) One could solve coupled Laplace equations with this jump in the gradient of $\phi$ as a constraint. This is not simply a matter of solving two Laplace equations with Neumann boundary conditions at the helix in the inner and outer regions, since only the jump in $\partial \phi / \partial r$ is known in advance. 
The additional constraint to be imposed is that $\phi(a)$ take on the same values in the two subdomains.

(ii) Alternatively, we may "smear" the surface charge by defining a charge density in the computational cell at the radius of the helix as $\rho=\sigma / \Delta r$, then solve a Poisson equation (including source terms from helix charge, beam particles, stray electrons, and any other sources):

$$
\nabla \cdot\left(\epsilon \nabla \phi^{1}\right)=-\rho^{1},
$$

where the "stencil" for cells with radial index $k$ corresponding to the helix radius might be:

$\frac{\epsilon_{\text {out }}\left(\phi_{k+1}^{1}-\phi_{k}^{1}\right)-\epsilon_{\mathrm{in}}\left(\phi_{k}^{1}-\phi_{k-1}^{1}\right)}{\Delta r^{2}}+\left(\frac{\partial^{2} \phi}{\partial z^{2}}\right)=-\frac{\sigma^{1}}{\Delta r}$.

For an infinitesimally thin sheet, the $\partial^{2} \phi / \partial z^{2}$ term is not needed since all $z$ variations are "slow" in comparison to jump in the radial electric field; but for a finite-thickness layer it is appropriate to include it.

(c) From the solution $\phi^{1}(r, z)$ to the Poisson equation, the electric field $E^{1}(r, z)$ can be obtained via finite differences, and the voltages $V^{1}(z)$ at the computational nodes on the helix obtained as the corresponding values of $\phi^{1}$.

(d) Time-advance the magnetic flux through each helix node $i$ using a finite-difference form of Eq. 6:

$$
\left(\Phi_{i}^{3 / 2}-\Phi_{i}^{1 / 2}\right) /(\Delta t)=-s\left(V_{i+1}^{1}-V_{i}^{1}\right) / \Delta z .
$$

(e) Obtain currents at the helix nodes from the magnetic fluxes by inverting the inductance matrix, Eq. 9 .

(de') Note that steps (d) and (e) may be combined, and introduction of the intermediary flux quantities $\Phi$ avoided, by substituting Eq. 9 into Eq. 14 to yield:

$$
\frac{\Delta z}{s} \frac{2 \pi a}{\Delta t} \sum_{j} M_{i j}\left(K_{z, j}^{3 / 2}-K_{z, j}^{1 / 2}\right)=\left(V_{i+1}^{1}-V_{i}^{1}\right),
$$

where the sum involving the $K_{z}^{3 / 2}$ is to be segregated into the left member and the matrix inverted. This is a dense matrix, with diminishing elements away from the diagonal.

This approach is especially attractive when the helix is terminated in a helical resistive line, so that the voltage drop per unit length has both inductive and resistive contributions in series (since the current in the inductor equals that in the resistor). The voltage drop between nodes $i+1$ and $i$ separated by a helix segment with a resistance per unit length $R_{i}$ has two contributions:

$$
\begin{aligned}
& \frac{\Delta z}{s} \frac{2 \pi a}{\Delta t} \sum_{j} M_{i j}\left(K_{z, j}^{3 / 2}-K_{z, j}^{1 / 2}\right) \\
& \quad+2 \pi a \Delta z R_{i} \frac{\left(K_{z, i}^{3 / 2}+K_{z, i}^{1 / 2}\right)}{2}=\left(V_{i+1}^{1}-V_{i}^{1}\right)
\end{aligned}
$$

(terms in $K_{z}^{3 / 2}$ are to be segregated into the left member).

(f) Use the resulting $K_{z, j}$ as sources to obtain $\mathbf{B}^{1}(r, z)$ or $\mathbf{B}^{3 / 2}(r, z)$, if necessary (when electron orbits are being computed, or for improved accuracy in ion orbits).
The "drive" can enter in any of several ways:

(i) If it is a current source, it replaces step (e) at node 1 using the prescribed input $I_{\text {input }}(t)$.

(ii) If it is a voltage source, it enters as an internal boundary condition in the Poisson solution of step (b).

(iii) If it is via a transformer primary, itself driven by a current source, it becomes an extra "node 0" in the mutual inductance matrix.

\section{DISCUSSION}

The equations presented herein define a detailed onedimensional model of wave propagation on the helix that can be coupled with a one-dimensional particle-in-cell model. The simplicity of such a model is attractive for development of insight and for rapid scoping studies; indeed, the simpler circuit model presented earlier lent valuable insight into the behavior of this novel system.

The circuit model from that earlier note was adapted for use in Warp, which allows multi-dimensional (2-D and 3D) particle-in-cell simulations to be carried out, including detailed space charge fields. Since an improved model is desired for design and analysis studies, we hope to implement this improved field model in Warp.

\section{ACKNOWLEDGMENTS}

The authors wish to acknowledge useful discussions with J-L. Vay and S. S Yu.

\section{REFERENCES}

[1] R. J. Briggs, "Pulse line ion accelerator concept," PRST-AB 9, 060401 (2006).

[2] R. J. Briggs, et al., Proc. 2005 Part. Accel. Conf.

[3] A. Friedman, et al., Proc. 2005 Part. Accel. Conf.

[4] G. Caporaso, et al., Proc. 2005 Part. Accel. Conf.

[5] W. Waldron, et al., Proc. 2005 Part. Accel. Conf.

[6] A. Friedman, "Studies of the Pulse-Line Accelerator Using a Circuit Model," available as LLNL Report UCRL-TR210492 (2005) or LBNL Report LBNL-58938 (2005).

[7] S. D. Nelson, et al., Proc. 2005 Part. Accel. Conf.

[8] W. L Waldron, et al., "Studies of the Pulse Line Ion Accelerator," these Proceedings.

[9] E. Henestroza, et al., "Electromagnetic Simulations of LBNL Pulse Line Ion Accelerator (PLIA) Experiments," these Proceedings.

[10] A. Friedman, "A Scaled Helix for Breakdown Studies," LLNL Report UCRL-TR-224518-REV-1 (2006).

[11] K. S. Yee, IEEE Trans. Antennas Prop. 14, 302-307 (1966).

[12] R. P. Feynman, R. B. Leighton, and M. L. Sands, The Feynman Lectures on Physics, p. 17-9 ff., Addison-Wesley, Reading, MA, 1964. 